\title{
SYNTHESIS OF ACYLGLYCERIDES WITH HIGH NUTRITIONAL VALUE BY AN ENZYMATIC TWO-STEP PROCESS
}

\author{
D.A. SANCHEZ ${ }^{\dagger, \S}$, G.M. TONETTO ${ }^{\dagger, \S}$ and M.L. FERREIRA ${ }^{\ddagger}, \S$ \\ † Departamento de Ingeniería Química, Universidad Nacional del Sur (UNS) \\ † Departamento de Química, Universidad Nacional del Sur (UNS) \\ $\S$ Planta Piloto de Ingeniería Química - PLAPIQUI (UNS - CONICET) \\ Bahia Blanca.Argentina.dsanchez@plapiqui.edu.ar
}

\begin{abstract}
D Acylglycerides are esters formed from glycerol and fatty acids. The structure of these glycerides affects their digestion and absorption. In this work, the synthesis of acylglycerides with high nutritional value by an enzymatic process in two stages is presented. In the first stage, the esterification of glycerol and capric acid catalyzed by Lipozyme RM IM was carried out. In this reaction, $73 \%$ conversion of capric acid and $71 \%$ selectivity to 1,3 -dicaprin was achieved. The diglyceride was separated and purified efficiently by a simple liquid-liquid extraction procedure. The dicaprin was esterified with palmitic acid using Burkholderia cepacia lipase immobilized on chitosan as the catalyst. In this second stage conversions of 1,3-dicaprin with values between 48 and $87 \%$ and with high selectivity towards the esterification of the sn-2 position were achieved. The reaction product had between 76 and $90 \%$ of acylglycerides with high nutritional value, depending on the reaction conditions.
\end{abstract}

Keywords - nutritional acylglycerides, enzymatic synthesis, lipases.

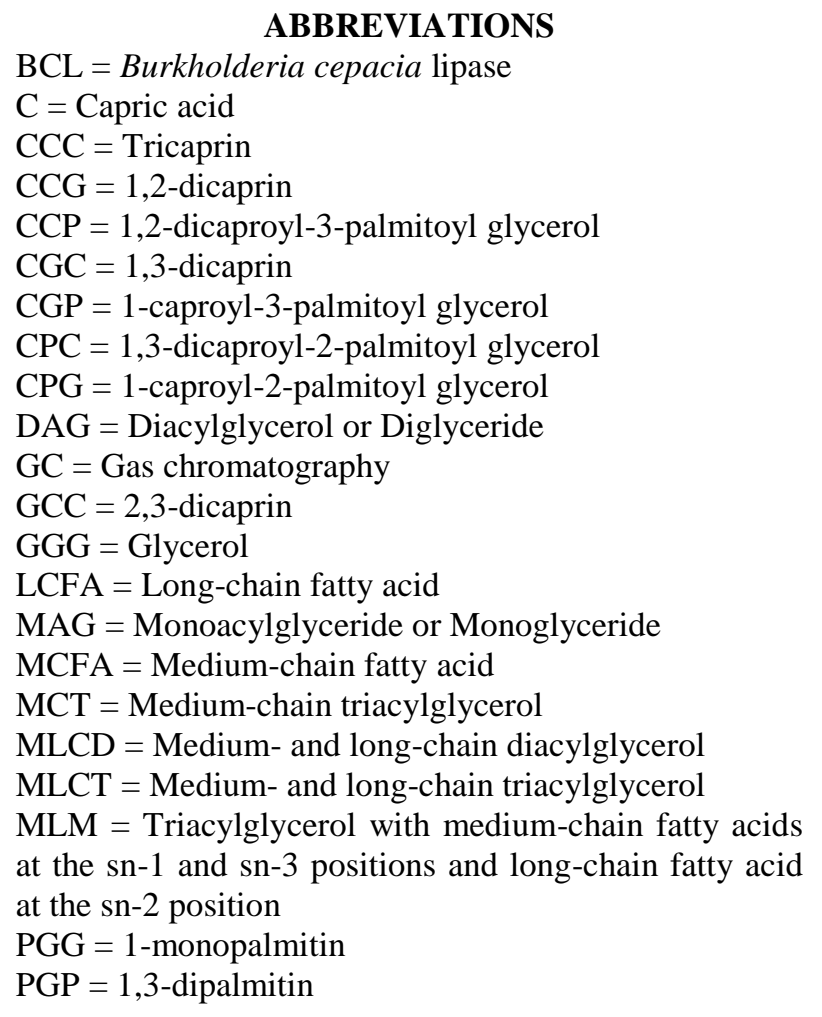

PPG $=1,2$-dipalmitin

PPL $=$ Porcine pancreas lipase

TAG $=$ Triacylglycerol or Triglyceride

\section{INTRODUCTION}

Triacylglycerols (TAG) are the main source of energy within food lipids. Medium-chain TAGs (MCT or MMM, where $\mathrm{M}$ is a fatty acid with a chain length of C6 to $\mathrm{C} 12$ ) have been employed as a rapid energy source due to their quick absorption, especially in infants with malabsorption and in child care (Mascioli et al., 1988). It is also used as an alternative solution for consumers with digestive problems (Lai et al., 2005). MCTs have no toxicological properties when their consumption is less than $30 \mathrm{~g}$ per day (Marten et al., 2006). However, MCT are not suitable for cooking due to its low smoke point and foam generation (Matsuo et al., 2001). To solve this problem, long-chain fatty acids (LCFA) were incorporated into MCT. The triglycerides containing in the same molecule medium chain fatty acids and long chain fatty acids have been defined as medium- and long-chain triglycerides (MLCT). These triglycerides are similar biochemically and physiologically to MCT (Kasai et al., 2003), with the advantage that they can be used as cooking oils for therapeutic purposes (Ogawa et al., 2007; Shinohara et al., 2005).

A particular case of MLCT are MLM-type triglycerides. They contain MCFA at the sn- 1 and sn-3 positions and LCFA at the sn-2 position. These triglycerides showed metabolic benefits compared to natural triglycerides or those produced with random structure (Christensen et al., 1995a, b). The MLM-type triglycerides have been synthesized using different methods (Bornscheuer et al., 2002; Xu, 2000, Akoh et al., 1995; Fomuso and Akoh, 1998; Shimada et al., 1996; Soumanou et al., 1997). However, the yields achieved were low and a large number of unwanted products were obtained with these synthesis methodologies. Obtaining MLM-type triglycerides from the esterification of the sn-2 position of 1,3-diglycerides (1,3-DAG) is an alternative but poorly studied pathway (Blasi et al., 2009; Wongsakul et al., 2004; Zhang et al., 2009).

On the other hand, diglycerides (DAG) are found in low proportions in oils and fats (levels below 5\%) (D'Alonzo et al., 1982). Several studies have reported nutritional benefits associated with the consumption of DAG, particularly 1,3-DAG. The incorporation in the diet of these diglycerides have allowed to reduce the concentration of triglycerides in serum and thereby re- 
duce visceral fat and body weight (Nagao et al., 2000; Watanabe et al., 1997; Murata et al., 1994).

In this work, the enzymatic synthesis of acylglycerides with high nutritional value was carried out through an enzymatic process in two stages.

\section{A. Materials}

\section{METHODS}

Lipozyme RM IM, which is a commercial form of the 1,3-specific lipase from $R$. miehei immobilized by adsorption on a macroporous anion exchange phenolic resin Duolite A-568, was kindly provided by Novo Nordisk A/S (Brazil). Burkholderia cepacia lipase was a generous donation from Amano Enzyme Inc. (Nagoya, Japan). Porcine pancreas lipase was provided by SigmaAldrich.

Glycerol, n-heptane and silica gel were supplied by Cicarelli Laboratorios. Capric acid, 1,2,4-butanetriol, tripalmitin and silylation reagents were obtained from Fluka. Monocaprin, dipalmitin, tricaprylin, tricaprin, trilaurin and trimyristin were supplied by Sigma-Aldrich. Absolute ethanol was supplied by Dorwil. Phenolphthalein, potassium hydroxide and pyridine were purchased from Anedra S.A., and chitosan was provided by Primex S.A. (Iceland). All the products were of analytical grade.

\section{B. Synthesis of 1,3-Dicaprin}

1,3-dicaprin (CGC) was obtained by esterification of glycerol (GGG) with capric acid (C) catalyzed by Lipozyme RM IM under previously optimized conditions (Sánchez et al., 2014a). The reaction was carried out in $10 \mathrm{~mL}$ vials kept in a bath with temperature control and magnetic stirring. For the reaction, $110 \mathrm{mg}$ of capric acid were dissolved in $3 \mathrm{~mL}$ of $\mathrm{n}$-heptane, then $250 \mathrm{mg}$ of glycerol adsorbed on $500 \mathrm{mg}$ of silica gel was added. The temperature was $60{ }^{\circ} \mathrm{C}$ and the reaction started with the addition of $10 \mathrm{mg}$ of Lipozyme RM IM. After 3 hours of reaction, another $10 \mathrm{mg}$ of the biocatalyst was added. The total reaction time was 6 hours. A $50 \mu \mathrm{L}$ sample was taken for analysis by gas chromatography (GC) and the remaining content was used to evaluate the acid conversion by acid-base titration with an ethanolic solution following a previously published methodology (Sánchez et al., 2014a).

\section{Separation and Purification of 1,3-Dicaprin}

After the esterification reaction was complete, the solids were separated by filtration. Monocaprin, dicaprin, tricaprin and unreacted capric acid were dissolved in nheptane. The capric acid not consumed in the reaction was neutralized and removed with $3 \mathrm{~mL}$ of an aqueous $0.1 \mathrm{M} \mathrm{KOH}$ solution. The mixture was stirred vigorously and then centrifuged at $8000 \mathrm{rpm}$ for $15 \mathrm{~min}$. The heptane phase was recovered and the procedure repeated. The final composition of the non-polar phase was determined by GC.

An equal volume of an ethanol/water mixture (90/10 $\mathrm{v} / \mathrm{v})$ was added to the n-heptane solution containing the acylglycerides. The sample was subjected to intense agitation and then centrifuged for $20 \mathrm{~min}$ at $8000 \mathrm{rpm}$. The phases were separated and the heptane phase was analyzed by GC. The extraction procedure was repeated three times (Fig. 2), finally the hydroethanolic solutions were combined and the solvent evaporated to recover the diglyceride.

The selection of solvents for liquid-liquid extraction was carried out using software for the molecular design of solvents. The results of the evaluation of solvents were previously published (Sanchez et al., 2014b) and the separation methodology could be used to separate other di- and triglycerides different from dicaprin and tricaprin.

\section{Immobilization of Burkholderia cepacia lipase}

Burkholderia cepacia lipase (BCL) was immobilized by physical adsorption on chitosan. For this purpose, 500 $\mathrm{mg}$ of commercial BCL powder were dispersed in 50 $\mathrm{mL}$ of twice-distilled water with $\mathrm{pH}=6.5$. The solution was stirred with a glass rod and then centrifuged to clarity. The supernatant was removed and placed in contact with $1 \mathrm{~g}$ of chitosan in a close beaker. Immobilization was carried out for $1 \mathrm{~h}$ with magnetic stirring at 400 $\mathrm{rpm}$ and at $35{ }^{\circ} \mathrm{C}$. The mixture was filtered to remove the solid, which was dried for $15 \mathrm{~h}$ at $30^{\circ} \mathrm{C}$ and then 24 $\mathrm{h}$ at $45{ }^{\circ} \mathrm{C}$ to remove the water adsorbed on the chitosan.

The obtained biocatalyst was placed in a beaker with $50 \mathrm{~mL}$ of distilled water and magnetically stirred at 400 rpm for $1 \mathrm{~min}$. This washing was carried out twice to remove the weakly adsorbed lipase. Subsequently the solid was dried as indicated above. The characterization of the biocatalyst obtained has been previously published (Sánchez et al., 2017).

\section{E. Esterification of Dicaprin with Palmitic Acid}

Dicaprin synthesized and purified by the procedures described was composed of $80 \%$ 1,3-dicaprin and $20 \%$ 1,2-dicaprin (80:20) (Sánchez et al., 2014a, b). The esterification reaction was performed in $10 \mathrm{~mL}$ vials, which were kept in a thermostatic bath with temperature control and magnetic stirring. The reaction time was 6 $\mathrm{h}$, and it was carried out as follows: $32 \mathrm{mg}$ of dicaprin (80:20) were dissolved in $2 \mathrm{~mL}$ of $\mathrm{n}$-heptane, then the given amount of palmitic acid for each reaction under study was added. When the reactant mixture reached the selected temperature, the reaction was started by adding the biocatalyst. The choice of solvent was performed according to $\mathrm{Bi}$ et al. (2015). Solvents with a $\log \mathrm{P}$ value higher than 4.0 increased the degree of esterification at the sn-2 position. The values of temperature (T), palmitic acid/dicaprin molar ratio $\left(\mathrm{R}_{\mathrm{M}}\right)$, and biocatalyst loading (B) were established according to a $2^{3}$ factorial design with two central points and a total of 10 experiments.

\section{F. Analysis of Samples by Gas Chromatography}

Samples were diluted with pyridine and siliylated with $N$-methyl- $N$-(trimethylsilyl) trifluoroacetamide (MSTFA). The analysis of the samples was performed in a Perkin Elmer AutoSystem XL gas chromatograph equipped with on-column injection, a flame ionization detector (FID) and a high temperature ZB-5HT Inferno 
capillary column $(15 \mathrm{~m} \times 0.32 \mathrm{~mm}$, with an ID of 0.10 $\mu \mathrm{m})$, using $\mathrm{H}_{2}$ as carrier gas. The detector temperature was maintained at $380{ }^{\circ} \mathrm{C}$. The initial column temperature was held at $50{ }^{\circ} \mathrm{C}$ for $1 \mathrm{~min}$, increased to $180{ }^{\circ} \mathrm{C}$ at a rate of $15{ }^{\circ} \mathrm{C} / \mathrm{min}$, then increased to $230{ }^{\circ} \mathrm{C}$ at 7 ${ }^{\circ} \mathrm{C} / \mathrm{min}$, further increased up to $370{ }^{\circ} \mathrm{C}$ at $10{ }^{\circ} \mathrm{C} / \mathrm{min}$, and finally maintained at $370{ }^{\circ} \mathrm{C}$ for $5 \mathrm{~min}$. Reported results are the average of two injections with an average relative error lower than $2 \%$. The determination of the elution times of the reactants and products was performed with high purity standards. Elution times for triglycerides without high purity standard were identified using another commercial standard of triglycerides with similar molecular weight (the commercial source of the standards is indicated in the Materials section).

\section{G. Identification of the Fatty Acid at sn-2 Position}

Detection of the fatty acid located at the sn- 2 position of the glycerol backbone was carried out by hydrolysis of acylglycerides using lipase from porcine pancreas (PPL) as catalyst, following the widely reported methodology (Miura et al., 1999; Schmid et al., 1999; Senanayake et al., 2002) combined with gas chromatography.

Free fatty acids were previously neutralized with 2 $\mathrm{mL}$ of an aqueous solution of $\mathrm{KOH} 0.1 \mathrm{M}$, and the phase containing acylglycerides was recovered (procedure described in Sánchez et al., 2014b. The solvent was evaporated and $1.9 \mathrm{~mL}$ of tris-hydrochloric buffer $(1.0 \mathrm{M}, \mathrm{pH}$ 8.0) and $0.1 \mathrm{~mL}$ of calcium chloride solution $(220 \mathrm{~g} / \mathrm{L})$ were added to the emulsified mixture. The reaction was carried out at $30{ }^{\circ} \mathrm{C}$ with magnetic stirring at $400 \mathrm{rpm}$ for $30 \mathrm{~min}$, and catalyzed with $20 \mathrm{mg}$ of PPL. Porcine pancreas lipase is recognized as 1,3-specific. The hydrolysis reaction generates 2-monoglycerides (2-MAG) and free fatty acids. The short reaction time allows avoiding the acyl migration reaction and correctly identifying the generated monoglycerides. However, the triglyceride conversion is partial due to the short reaction time, the results are extrapolated for the total conversion. The identification of 2-MAG, free fatty acids and diglycerides obtained by this hydrolysis reaction makes possible to know the composition of the starting sample.

\section{A. Synthesis of 1,3-Dicaprin}

III. RESULTS AND DISCUSSION

Under the evaluated reaction conditions, the conversion of capric acid was $73 \%$. The final product was composed of $18 \%$ monocaprin, $72 \%$ dicaprin and $9 \%$ tricaprin (in molar percentage). Of the total dicaprin generated, 93\% was 1,3 dicaprin (CGC), 5\% was 1,2dicaprin (CCG) and $2 \%$ was 2,3-dicaprin (GCC). The selectivity to 1,3 -dicaprin was $71 \%$. A typical chromatogram of the reaction products and unreacted substrates is shown in Fig. 1.

\section{B. Separation and Purification of Dicaprin}

The product of the esterification reaction was composed of monocaprin, dicaprin, tricaprin and the unconsumed fatty acid. All the compounds were dissolved in the reaction solvent (n-heptane).

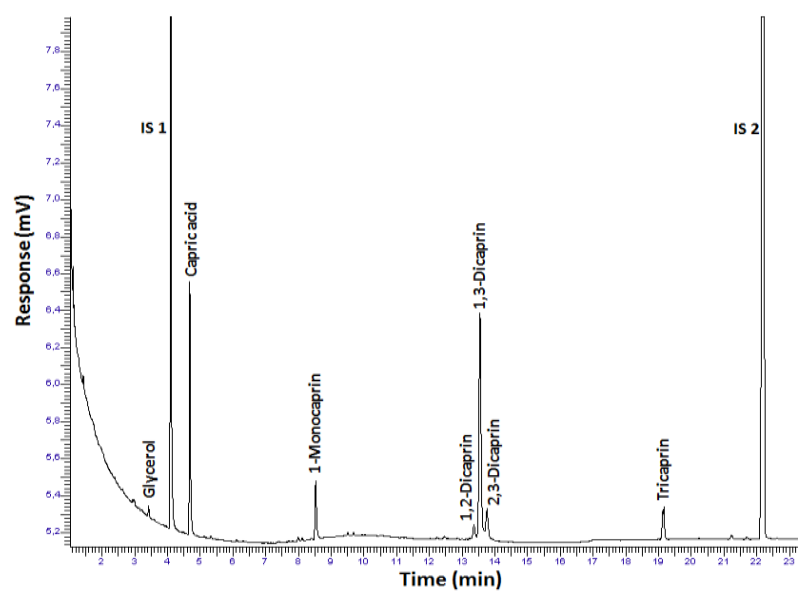

Fig. 1. Typical chromatogram of the reaction products and unconsumed reactants in the enzymatic esterification of glycerol and capric acid using Lipozyme RM IM. IS1 and IS2: Internal calibration standards.

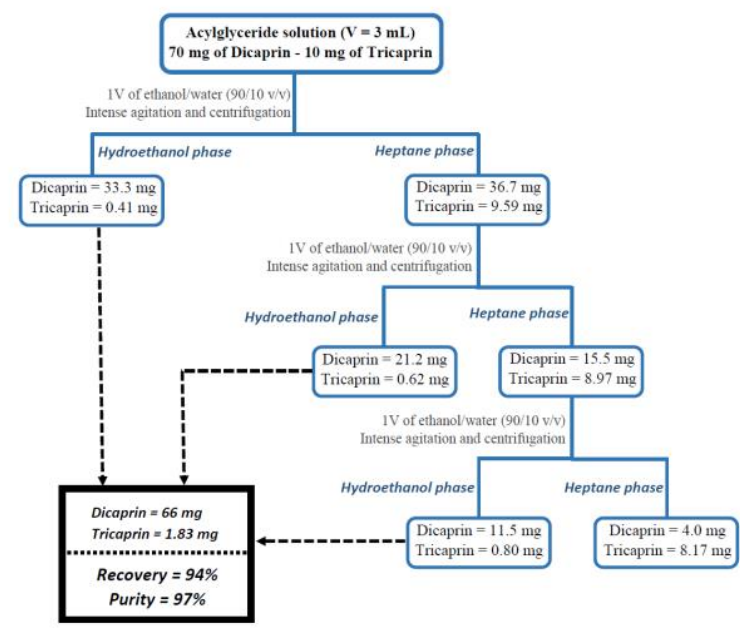

Fig. 2. Results of the liquid-liquid extraction process.

The unreacted fatty acid was neutralized with an aqueous $0.1 \mathrm{M} \mathrm{KOH}$ solution. The neutralization and removal of the acid were performed by two washes with the aqueous solution. With this simple procedure, it was possible to remove $99.5 \%$ of the unreacted fatty acid. In addition, the aqueous solution was able to extract $95 \%$ of the monocaprin generated in the reaction. During the neutralization process, losses of dicaprin and tricaprin of 4.5 and $4 \%$ respectively occurred.

The liquid-liquid extraction process in three stages, shown in Fig. 2, allowed recovering $94 \%$ of the initial dicaprin with a purity of $97 \%$.

\section{Synthesis of Acylglycerides with High Nutritional Value}

Using a factorial design, the reaction conditions for the esterification of dicaprin (80:20) with palmitic acid were evaluated. The reaction was catalyzed by Burkholderia cepacia lipase immobilized by physical adsorption on chitosan.

Esterification of 1,3-dicaprin generated 1,3dicaproyl-2-palmitoyl glycerol (CPC), a MLM-type triglyceride with high nutritional value. Furthermore, the 
esterification of 1,2-dicaprin allowed the generation of 1,2-dicaproyl-3-palmitoyl glycerol (CCP), a TCML that is also valuable from a nutritional point of view.

In addition to the esterification reaction, the hydrolysis reaction was also important in the system under study. This secondary reaction generated free capric acid and even glycerol. In addition, the products of the hydrolysis were re-esterified generating different acylglycerides, such as: 1-monopalmitin (PGG), 1,2dipalmitin (PPG), 1,3-dipalmitin (PGP), 1-caproyl-2palmitoyl glycerol (CPG), 1-caproyl-3-palmitoyl glycerol (CGP), tricaprin (CCC) and 1-caproyl-2,3palmitoyl glycerol (CPP). Several of them are nutritionally valuable: CCC is a rapidly metabolizable TCM, CGP is a diglyceride of type 1,3-DAG, CPG is a diglyceride with metabolic characteristics similar to a MLMtype triglyceride but with reduced calories.

A typical chromatogram of the products of the esterification reaction and unreacted substrates is shown in Fig. 3.

Free fatty acids and glycerol were removed by performing two washes with a $0.1 \mathrm{M}$ aqueous solution of $\mathrm{KOH}$.

Table 1 shows the factors and levels evaluated in the factorial design. The conversion of CGC and the mole fraction of valuable acylglycerides in the reaction prod- uct are presented as the response variables. CPC, CCP, CCC, CPG, and CGP were considered as the valuable acylglycerides. In addition, unreacted 1,3-dicaprin is also interesting from a nutritional point of view.

Second order models were used to adjust the data. These models were refined by applying the F-value test (F-test), also called the Fisher-Snedecor test (Besset, 2001). Equation 1 represents the quadratic model with all its variables and combinations of them.

$$
\begin{aligned}
X_{A c}= & A_{0}+A_{1} B+A_{2} T+A_{3} R_{M}+A_{4} B T+A_{5} B R_{M}+ \\
& A_{6} T R_{M}+A_{7} B^{2}+A_{8} T^{2}+A_{9} R_{M}^{2}
\end{aligned}
$$

where $R_{M}$ is the palmitic acid/dicaprin molar ratio, $T$ is the reaction temperature, $B$ is the biocatalyst mass, and $A_{i}$ are the regression coefficients of the model.

In Table 1a, only the reaction products were considered. The final mixture was composed of between 48 and $68 \%$ of nutritionally interesting acylglycerides. The generation of nutritionally valuable acylglycerides was favored by the increase of the biocatalyst mass and the temperature. The concentration of palmitic acid did not have a statistically significant effect on this response. The relationship between the molar fraction of these acylglycerides (excluding 1,3-dicaprin) and the studied factors was appropriately adjusted by Eq. 2 with $\mathrm{R}^{2}=$ $97.4 \%$.

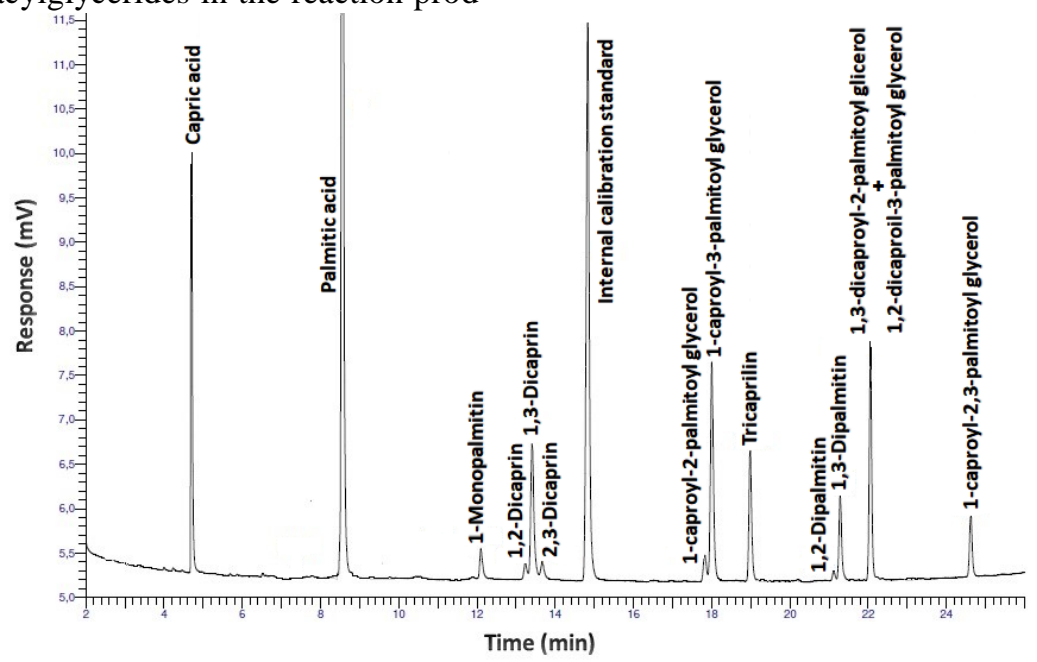

Fig. 3. Typical chromatogram of the reaction products and unconsumed reactants for the enzymatic esterification of dicaprin and palmitic acid. Experimental conditions: dicaprin $=32 \mathrm{mg}, \mathrm{R}_{\mathrm{M}}=3 \mathrm{~mol} / \mathrm{mol}$, temperature $=40^{\circ} \mathrm{C}$, and biocatalyst load $=150 \mathrm{mg}$. Reaction time: $6 \mathrm{~h}$.

Table 1. Experimental factors and parameters evaluated during the enzymatic esterification of dicaprin with palmitic acid catalyzed by Burkholderia cepacia lipase immobilized on chitosan at $6 \mathrm{~h}$ of reaction.

\begin{tabular}{ccc|c|c|c}
\hline \multicolumn{3}{c|}{ Experimental factors } & $\begin{array}{c}\text { 1,3-dicaprin } \\
\text { conversión }(\%)\end{array}$ & $\begin{array}{c}\text { Valuable acylglycerides } \\
(\text { molar \%) }\end{array}$ & $\begin{array}{c}\text { Valuable acylglycerides } \\
(\text { molar \%) }\end{array}$ \\
\cline { 1 - 3 } $\mathrm{B}(\mathrm{mg})$ & $\mathrm{T}\left({ }^{\circ} \mathrm{C}\right)$ & $\mathrm{R}_{\mathrm{M}}(\mathrm{mol} / \mathrm{mol})$ & 90 & 68.1 & 80.9 \\
50 & 60 & 1 & 68 & 54.6 & 88.4 \\
50 & 60 & 3 & 60 & 52.4 & 89.2 \\
150 & 40 & 1 & 81 & 62.1 & 81.6 \\
50 & 40 & 3 & 50 & 47.8 & 90.0 \\
150 & 60 & 3 & 89 & 65.9 & 76.2 \\
100 & 50 & 2 & 81 & 60.5 & 81.0 \\
150 & 40 & 3 & 84 & 64.5 & 79.5 \\
100 & 50 & 2 & 83 & 62.4 & 81.2 \\
50 & 40 & 1 & 54 & 49.7 & 90.3 \\
\hline
\end{tabular}

a Acylglycerides with high nutritional value without considering the presence of 1,3-dicaprin in the final product.

${ }^{\mathbf{b}}$ Acylglycerides with high nutritional value taking into account unreacted 1,3-dicaprin in the final product. 


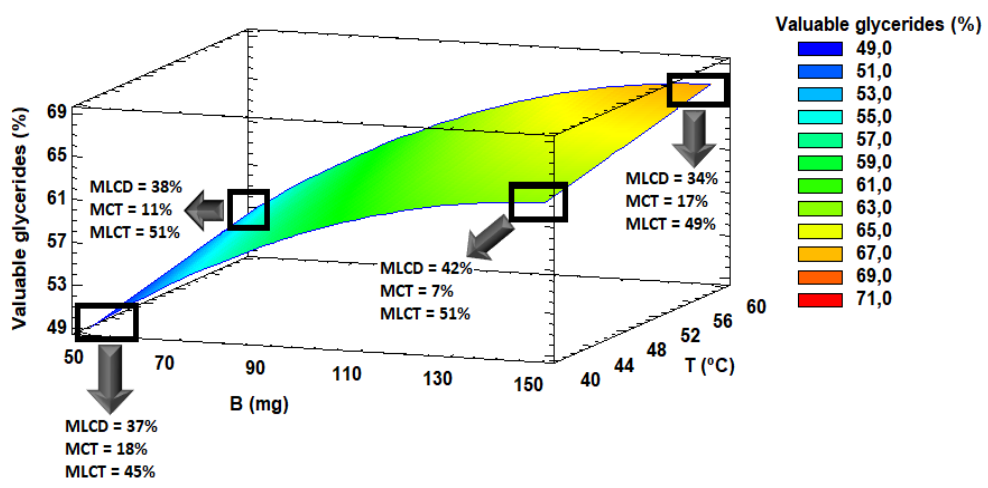

Fig. 4. Molar fraction of nutritionally valuable acylglycerides obtained from the esterification of dicaprin with palmitic acid catalyzed by the Burkholderia cepacia lipase immobilized on chitosan. Variables: mass of biocatalyst and reaction temperature. MLCD = medium- and long-chain diacylglycerides, MCT = medium-chain triacylglycerides, and MLCT = medium- and longchain triacylglycerides.

Valuable glycerides $=23.61+0.405 \mathrm{~B}+0.211 \mathrm{~T}-0.00133 \mathrm{~B}^{2}$

A surface plot for this response is presented in Fig. 4 , where the variation in the composition of the glycerides with nutritional value is shown as a function of the variation in the reaction conditions. The molar percentage of medium- and long-chain diglycerides (MLCD), MCT and MLCT are shown on the vertices of the graph. It is possible to observe the change in the composition of the final product as a function of the reaction conditions. In addition, it is evident that the formation of MLCD and MLCT was favored when the reaction was carried out with the highest biocatalyst load and the lowest reaction temperature (within the range of study).

1,3-Dicaprin is also a valuable compound from a nutritional point of view. The total conversion of this diglyceride was between 50 and $90 \%$. In this way, the presence of unreacted 1,3-dicaprin enriched the final product. Table $1 \mathrm{~b}$ shows the percentage of acylglycerides in the final product considering unreacted 1,3dicaprin as a nutritionally valuable acylglyceride. In this case, the final blend consisted of 76-90\% acylglycerides with high nutritional value.

Equation 3 relates the fraction of nutritionally valuable glycerides to the studied factors. This equation was obtained from multiple linear regression, and only the statistically significant effects were considered. The coefficient of determination $\left(\mathrm{R}^{2}\right)$ for this model was $99.7 \%$.

Valuable glycerides $=107-0.372 \mathrm{~B}+0.00137 \mathrm{~B}^{2}-0.0233 \mathrm{TR}_{\mathrm{M}}$

The synthesis of acylglycerol mixtures with different compositions (of high nutritional value) could be performed according to the requirements or a desired composition by modifying the reaction conditions.

\section{CONCLUSIONS}

The synthesis of acylglycerides with high nutritional value was efficiently carried out by an enzymatic process in two stages, including synthesis reactions and purification processes.

The final product was a mixture of acylglycerides formed mainly by glycerol esterified with capric acid at the sn- 1 and sn-3 positions and palmitic acid at the sn-2 position.

The variation in reaction conditions in the second stage allowed obtaining products with different acylglyceride composition. This process could be implemented for the production of nutritional glycerides with composition as required.

\section{ACKNOWLEDGMENTS}

The authors thank the Agencia Nacional de Promoción Científica y Tecnológica (National Agency of Scientific and Technological Promotion, Argentina) and the Consejo Nacional de Investigaciones Científicas y Técnicas (National Council for Scientific and Technological Research, CONICET) for the financial support.

\section{REFERENCES}

Akoh, C.C., B.H. Jennings, D.A. Lillard, "Enzymatic Modification of Trilinolein: Incorporation of n-3 Polyunsaturated Fatty Acids," J. Am. Oil Chem. Soc., 72, 1317-1321 (1995).

Besset, D.H., Object-oriented implementation of numerical methods: an introduction with Java and Smalltalk, Square Bracket Associates, Switzerland (2001).

Bi, Y.H., Z.Y. Wang, Z.Q. Duan, X.J. Zhao, X.M. Chen and L.H. Nie LH, "An insight into the solvent effect on the positional selectivity of the immobilized lipase from Burkholderia cepacia in 1,3-diolein synthesis," RSC Adv., 5, 23122-23124 (2015).

Blasi, F., S. Maurelli, L. Cossignani, G. D’Arco, M.S. Simonetti and P. Damiani, "Study of some experimental parameters in the synthesis of triacylglycerols with CLA isomers and structural analysis," $J$. Am. Oil Chem. Soc., 86, 531-537 (2009).

Bornscheuer, U.T., M. Adamczak and M.M. Soumanou, Lipase-Catalyzed Synthesis of Modified Lipids. Lipids as Constituents of Functional Foods, PJ Barnes \& Associates, Bridgwater, United Kingdom, 149-182 (2002).

Christensen, M.S., A. Müllertz and C.E. Høy, “Absorption of Triglycerides with Defined or Random Structure by Rats with Biliary and Pancreatic Diversion," Lipids, 30, 521-526 (1995a). 
Christensen, M.S., C.E. Høy, C.C. Becker and T.G. Redgrave, "Intestinal Absorption and Lymphatic Transport of Eicosapentaenoic (EPA), Docosahexaenoic (DHA), and Decanoic Acids: Dependence on Intramolecular Triacylglycerol Structure," Am. J. Clin. Nutr., 61, 56-61 (1995b).

D’Alonzo, R.P., W.J. Kozarek and R.L. Wade, “Glyceride Composition of Processed Fats and Oils As Determined by Glass Capillary Gas Chromatography," J. Am. Oil Chem. Soc., 59, 292-295 (1982).

Fomuso, L.B. and C.C. Akoh, "Structured Lipids: Lipase-Catalyzed Interesterification of Tricaproin and Trilinolein," J. Am. Oil Chem. Soc., 75, 405410 (1998).

Kasai, M., N. Nosaka, H. Maki, S. Negishi, T. Aoyama, M. Nakamura and K. Kondo, "Effect of dietary medium-and long-chain triacylglycerols (MLCT) on accumulation of body fat in healthy humans," Asia Pac. J. Clin. Nutr., 12, 151-160 (2003).

Lai, O.M., C.T. Low and C.C. Akoh, "Lipase-catalyzed acidolysis of palm olein and caprylic acid in a continuous bench-scale packed bed bioreactor," Food Chem., 92, 527-533 (2005).

Mascioli, E.A., V.K. Babayan, B.R. Bistrian and G.L. Blackburn, "Novel triglycerides for special medical purposes," J. Parenter. Enteral. Nutr., 12, 127S132S (1988).

Marten, B., M. Pfeuffer and J. Schrezenmeir, "Mediumchain triglycerides," Int. Dairy J., 16, 1374-1382 (2006).

Matsuo, T., M. Matsuo, N. Taguchi and H. Takeuchi, "The thermic effect is greater for structured medium-and long-chain triacylglycerols versus longchain triacylglycerols in healthy young women," Metabolism, 50, 125-130 (2001).

Miura, S., A. Ogawa and H. Konishi, "A rapid method for enzymatic synthesis and purification of the structured triacylglycerol, 1,3-dilauroyl-2-oleoylglycerol," J. Am. Oil Chem. Soc., 76, 927-931 (1999).

Murata, M., K. Hara and T. Ide, "Alteration by Diacylglycerols of the Transport and Fatty Acid Composition of Lymph Chylomicrons in Rats," Biosci. Biotechnol. Biochem., 58, 1416-1419 (1994).

Nagao, T., H. Watanabe, N. Goto, K. Onizawa, H. Taguchi and N. Matsuo N, "Dietary Diacylglycerol Suppresses Accumulation of Body Fat Compared to Triacylglycerol in Men in a Double-Blind Controlled Trial," J. Nutr., 130, 792-7 (2000).

Ogawa, A, N. Nosaka, M. Kasai, T. Aoyama, M. Okazaki, O. Igarashi and K. Kondo K, "Dietary medium-and long-chain triacylglycerols accelerate dietinduced thermogenesis in humans," J. Oleo Sci., 56, 283-287 (2007).

Sánchez, D.A., G.M. Tonetto and M.L. Ferreira, "Enzymatic synthesis of 1,3-dicaproyglycerol by esterification of glycerol with capric acid in an organic solvent system". J. Mol. Catal. B Enzym., 100, 718 (2014a).

Sánchez, D.A., G.M. Tonetto and M.L. Ferreira, "Separation of acylglycerides obtained by enzymatic esterification using solvent extraction," J. Am. Oil Chem. Soc., 91, 261-270 (2014b).

Sánchez, D.A., G.M. Tonetto and M.L. Ferreira, "Screening of lipases with unusual high activity in the sn-2 esterification of 1,3-dicaprin under mild operating conditions," J. Agric. Food Chem., 65, 5010-5017 (2017).

Schmid, U., U.T. Bornscheuer, M.M Soumanou, G.P. McNeill and R.D. Schmid, "Highly selective synthesis of 1,3-oleoyl-2-palmitoylglycerol by lipase catalysis," Biotechnol. Bioeng., 64, 678-684 (1999).

Senanayake, S.N. and F. Shahidi F, "Enzyme-catalyzed synthesis of structured lipids via acidolysis of seal (Phoca groenlandica) blubber oil with capric acid," Food Res. Int., 35, 45-752 (2002).

Shimada, Y., A. Sugihara, H. Nakano, T. Yokota T, Nagao, S. Komemushi and Y. Tominaga, "Production of Structured Lipids Containing Essential Fatty Acids by Immobilized Rhizopus delemar Lipase," $J$. Am. Oil Chem. Soc., 73, 1415-1420 (1996).

Shinohara, H, A. Ogawa, M. Kasai and T. Aoyama, "Effect of randomly interesterified triacylglycerols containing medium-and long-chain fatty acids on energy expenditure and hepatic fatty acid metabolism in rats". Biosci. Biotechnol. Biochem., 69, 1811-1818 (2005).

Soumanou M.M., U.T. Bornscheuer, U. Menge and R.D. Schmid, "Synthesis of Structured Triglycerides from Peanut Oil with Immobilized Lipase," J. Am. Oil Chem. Soc., 74, 427-433 (1997).

Watanabe, H., K. Onizawa, H. Taguchi, M. Kobori, H. Chiba, S. Naito, N. Matuo, T. Yasukawa, M. Hattori and H. Shimasaki, "Nutritional Characterization of Diacylglycerols in Rats," J. Oleo Science, 46, 301-307 (1997).

Wongsakul, S., H. Aran and U.T. Bornscheuer UT, "Lipase-catalyzed synthesis of structured triacylglycerides from 1, 3-diacylglycerides," J. Am. Oil Chem. Soc., 81, 151-155 (2004).

$\mathrm{Xu}, \mathrm{X}$., "Production of Specific-Structured Triacylglycerols by Lipase-Catalyzed Reactions: A Review," Eur. J. Lipid Sci. Tech., 1002, 287-303 (2000).

Zhang, H., G. Önal, C. Wijesundera and X. Xu, "Practical synthesis of 1,3-oleoyl 2-docosahexaenoylglycerol by lipase-catalyzed reactions: An evaluation of different reaction routes," Process Biochem., 44, 534-539 (2009).

Received February 26, 2018.

Sent to Guest Editor February 26, 2018.

Accepted February 11, 2019.

Recommended by Guest Editor Patricia M. Hoch 